\title{
Beneficial Role of Platelet -Rich Plasma (PRP) combined with Glass Wool Filtration for Best Recovery of Spermatozoa in Infertile Men
}

\author{
Watfaa A. Abduljabar, Hayder A. L. Mossa and Muayad S. Abood
}

\section{ABSTRACT}

Background: Platelet -Rich Plasma (PRP) is a novel therapeutic agent used in multiple medical fields and one of these fields is the reproduction for best spermatozoa preparation and activation for upgrading activity and motility of the spermatozoa and filtered with glass wool filtration to eliminate any round cells and leukocytes from the sample.

Objectives: The aim of this research is to study some sperm characteristics in two groups, the normozoospermic infertile men and th asthenozoospermic infertile group before and after activation with Glass wool Filtration and PRP and compare between both.

Patients and Methods: In this study 60 infertile men were enrolled and divided into 2 groups, 15 normozoospermic infertile men, and 45 ashenozoospermic infertile men during their attendance to the Infertility Clinic in High Institute for Infertility Diagnosis and Assisted Reproductive Technologies Al-Nahrain University. The collected semen samples, and seminal fluid analysis were assessed, each semen sample was divided into 3 tubes and labelled as the 1st tube which was before activation, the 2nd tube was after glass wool filtration activation technique and the 3rd tube was for semen activated by adding PRP to the medium used for glass wool filtration activation.

Results: Both techniques showed increased and improved sperm motility, but the PRP was superior to the glass wool alone in upgrading Sperm Grade A Motility \%, Sperm Grade B Motility \% and decreasing Sperm Grade C Motility \%, and Sperm Grade D Motility (Immotile Sperm).

Conclusion: The PRP was significantly effective in improving the sperm activity and upgrading sperm motility more than Glass Wool Filtration technique.

Keywords: Glass Wool Filtration (GWF), Platelet -Rich Plasma (PRP).

\section{INTRODUCTION}

Sub fertility and infertility are a common medical issue, regardless of the cause, a couple are considered to be infertile after failure to conceive following 12 months of regular unprotected sexual intercourse which is important determinant for the occurrence of pregnancy [1]. This condition affects approximately $10-15 \%$ of Reproductive age -group. Men have to be evaluated by seminal fluid analysis, hormonal assay and other investigations. In general infertility evaluation is initiated 12 months after regular unprotected sexual intercourse Causes of infertility could be congenital or acquired including Genital infections, autoimmune diseases, malignancies, scrotal disease, endocrine disease, genetic disorder and others. Assisted
Published Online: September 30,2021

ISSN: 2736-5476

DOI: 10.24018/ejclinicmed.2021.2.5.125

W. A. Abduljabar

High Institute for Infertility Diagnosis and Assisted Reproductive Technologies Al-Nahrain University, Baghdad, Iraq. H. A. L. Mossa*

High Institute for Infertility Diagnosis and Assisted Reproductive Technologies Al-Nahrain University, Baghdad, Iraq.

(e-mail: haydermossa@gmail.com)

M. S. Abood

High Institute for Infertility Diagnosis and Assisted Reproductive Technologies Al-Nahrain University, Baghdad, Iraq.

*Corresponding Author

Reproductive Technologies used to treat infertility includes the following: in vitro fertilization (IVF), Gamete intrafallopian transfer (GIFT). Zygote Intrafallopian Transfer (ZIFT), Intracytoplasmic Sperm Injection (ICSI)Intrauterine Insemination (IUI), Sperm-oocyteembryo cryopreservation [2].

Types of spermatozoa abnormalities.

1) Asthenozoospermia; is defined as $<40 \%$ sperm motility or less than $32 \%$ progressive motility [3], While in WHO 1999 is explained as the total sperm motility less than $50 \%$, it represents important cause of reduced fertility or infertility in men that decrease oocyte fertilization ability [4].

2) Azoospermia; is defined as a total absence of spermatozoa from at least two separate centrifuged 
semen specimens [5].

3) Oligozoospermia; is a term refers to a concentration less than $15 \mathrm{million} / \mathrm{ml}$ according to the WHO [6] many factors can cause oligospermia, such as varicocele, infections, ejaculation problems overheating testicles, drugs and alcohol use [7].

4) Teratozoospermia; is defined as the decrease in percentage of normal sperm morphology less than $4 \%$ using Kruger strict criteria WHO (2010) or $<30 \%$ WHO (1999), one of the indicators for DNA damage and other defects is the morphological abnormalities of the sperm [8].

Sperm Preparation Methods: Following the harvest of the classical swim- up technique by Mahadevan and Baker [9] abundant of sperm preparation techniques have been used to segregate a high motility spermatozoon; of these methods is:

Glass Wool Filtration (GWF), technique which had been described for the first time by Paulson and Polakoski in 1977 [10], it is easy to perform, and the spermatozoa resulted with good motility and specimens with decrease sperm density ejaculates can be separated [11], separation of motile spermatozoa is performed by densely packed glass wool fibers [12].

Platelet-Rich Plasma(PRP), is a novel therapeutic option that is used in multiple medical fields and one of these fields is the Reproduction, PRP is defined as an autologous concentration of human platelets that is 3-5 times greater than the physiological concentration of thrombocytes in whole blood [13], the inclusion of the PAF (Platelet Activating Factor) to the IUI sperm wash was a procedure significantly improves pregnancy rates, the significant improvement affect more the men with normal semen parameters [14].

\section{PATIENTS AND METHODS}

The study included 60 infertile men were enrolled in this study, they were divided into two groups, (forty-five (45) as asthenozoospermic, and fifteen (15) as normozoospermic subjects) during their attendance to the infertility clinic at the High Institute for Infertility Diagnosis and Assisted Reproductive Technologies, Al- Technologies Al-Nahrain University from the period October 2019 till August 2020. The study included these two groups and merged them within infertile group to mimic the best sperm activation outcome and exclude any relevant opposing factor. The seminal fluid analysis was assessed, and each semen sample was divided into 3 portions; the first part was labelled for sperm characteristics before activation which was $0.5 \mathrm{ml}$, the second portion $1 \mathrm{ml}$ was labelled for sperm characteristic after Glass wool filtration, the third portion $1 \mathrm{ml}$ was labelled for sperm characteristics following PRP activation. For the second portion the semen was prepared first with washing by $1 \mathrm{ml}$ FertiCult Flushing media and centrifugation $3000 \mathrm{rpm}$ for 5 minutes then discard the supernatant, followed by another $1 \mathrm{ml}$ of FertiCult Flushing media with centrifugation again $4000 \mathrm{rpm}$ for 10 minutes followed by incubation for 30 minutes, then filtration by the glass wool method. For the third portion the same previous steps were followed by adding Platelet -rich plasma after preparing it with a percent of $2 \%$, added to the specimen followed by incubation at $37^{\circ} \mathrm{C}$ for one hour since the Filtration by Glass Wool, then, from each sample, a drop of $10 \mu 1$ was aspirated and put on a slide with a coverslip and was examined under microscope at the $400 \mathrm{x}$ objective to assess the sperm parameters.

\section{RESUlts}

The seminal fluid characteristics of infertile men after activation, both Glass Wool Filtration and PRP, resulted in a highly significant increase in mean Sperm Grade A Motility percent and Grade B Motility \% $(\mathrm{P}<0.001)$, in comparison with before activation, however the PRP resulted in more significant increase, added to that both Glass Wool and PRP resulted in highly significant reduction in mean Grade $\mathrm{C}$ Motile Sperm \% and Grade D Immotile Sperm \% $(\mathrm{P}<0.001)$ in comparison with before activation, however the PRP resulted in more significant reduction.

TABLE I: BEFORE -AND AFTER ACTIVATION WITH GLASS WOOL FILTRATION AND PRP TECHNIQUES OBTAINED SPERM PARAMETERS COMPARISON

\begin{tabular}{ccccc}
\hline \hline Characteristic & $\begin{array}{c}\text { Before } \\
\text { activation }\end{array}$ & $\begin{array}{c}\text { Glass } \\
\text { wool }\end{array}$ & PRP & $p$ \\
\hline \hline Concentration & 42.02 & 34.72 & 33.17 & $<0.001$ \\
million /ml & \pm 9.79 & \pm 7.61 & \pm 6.53 & $\mathrm{Ph}$ \\
Grade A Motility & $5.60 \pm 6.50$ & 18.75 & 31.37 & $<0.001$ \\
$\%$ & $\mathrm{C}$ & \pm 12.06 & \pm 7.95 & $\mathrm{Ph}$ \\
& & $\mathrm{B}$ & $\mathrm{A}$ & $\mathrm{HS}$ \\
Grade B Motility & 28.63 & 52.05 & 58.25 & $<0.001$ \\
$\%$ & \pm 5.66 & \pm 9.01 & \pm 7.43 & $\mathrm{Ph}$ \\
& $\mathrm{C}$ & $\mathrm{B}$ & $\mathrm{A}$ & $\mathrm{HS}$ \\
Grade A and B & 34.32 & 70.72 & 89.62 & $<0.001$ \\
Motility \% & \pm 9.57 & \pm 11.43 & \pm 4.18 & $\mathrm{Ph}$ \\
Grade C Motility & 27.63 & 13.50 & 5.32 & $<0.001$ \\
$\%$ & \pm 8.27 & \pm 5.41 & \pm 2.20 & $\mathrm{Ph}$ \\
Grade D & $\mathrm{A}$ & $\mathrm{B}$ & $\mathrm{C}$ & $\mathrm{HS}$ \\
(Immotile & \pm 1.83 & 15.33 & 5.07 & $<0.001$ \\
sperm) \% & $\mathrm{A}$ & \pm 7.48 & \pm 2.23 & $\mathrm{Ph}$ \\
Morphologically & 40.22 & 58.63 & 67.57 & $<0.001$ \\
Normal Sperm & \pm 9.37 & \pm 5.78 & \pm 2.00 & $\mathrm{Ph}$ \\
$\%$ & $\mathrm{C}$ & $\mathrm{B}$ & $\mathrm{A}$ & $\mathrm{HS}$ \\
\hline \hline
\end{tabular}

Data were represented as mean \pm standard deviation. Ph. Phillais multiple repetition test. HS; highly significant at $(\mathrm{P} \leq 0.001)$, capital letters $\mathrm{A}, \mathrm{B}$ and $\mathrm{C}$ were used to show the level of significance between two successive groups, so that different letters indicate significant difference while similar letters indicate no significant difference.

\section{DISCUSSION}

Results of Assisted Reproductive Technologies (ART's) still do not exceed high percentages due to different factors, one of them is male factor infertility. Therefore, the global companies concern on the culture media, combinations and techniques that used for activation of semen samples of infertile men. However, till now, there are many semen samples did not response to sperm preparation techniques leading to failure of sperm activation [13].

Platelets as a main component of the PRP, contain more than 1100 different proteins, with numerous posttranslational modifications, resulting in over 1500 proteinbased bioactive factors [14]. These factors include immune system messengers, growth factors, enzymes and their 
inhibitors and other factors which can participate in tissue repair and wound healing. Another important characteristic of PRP is that represents an autologous product, which is prepared from the patient's own blood. Therefore, the use of autologous PRP eliminates any concerns about the risk of crossed contamination, disease transmission or immune reactions [15].

There is important evidence to introduce Plasma-Rich platelet in many aspects of Reproduction, its therapeutic effect is due to the presence of multiple growth factors such as (TGF- $\beta$ ) Transforming growth factor- $\beta$, fibroblast growth factor (FGF), platelet derived growth factors (PDGF), and others. The inclusion of these growth factors to the intrauterine Insemination (IUI) trend and sperm washing preparation within sperm activation significantly improves pregnancy rate [16]. Platelet activating factor (PAF) is present in human spermatozoa and its endogenous content has a positive relationship to sperm motility and pregnancy rates [17].

In this current study, which is the first one within this trend, so there were no previous studies to compare the sperm activation outcome with them.

The inclusion of PRP as activating medium with Glass Wool Filtration significantly had showed significant upgrading the sperm motility and morphologically normal sperm $\%$.

From the results of this study it can conclude that both Glass wool and PRP are effective in reducing semen concentration and upgrading sperm motility, PRP is more superior than the Glass Wool in improving the sperm Quality and Morphology and significantly improve Grade A sperm motility \% and improving Grade B motile sperm \%, both methods reduce Grade $\mathrm{C}$ motile sperm \% and Grade D immotile, and in minimizing the round cell count and leukocytes but PRP is superior in its effect.

\section{REFERENCES}

[1] S. S. Al-Dujaily, H. A. L. Mossa and S. N. Alwachi, "Study of Calpain Activity, Sperm Penetration Assay in Unexplained Infertile Men in Relevance to Assisted Reproductive Technologies," World Journal of Pharmaceutical Research, 4(2):100-117, 2015.
[2] E. E Puscheck and R. S. Lucidi, "Infertility Practice, Essential Overview," Etiology of Infertility, 25, 2020.

[3] S. Barak and H. W. Gordon Baker, "Clinical management of male infertility," Endocrinology: Adult and Pediatric. 7th ed. Philadelphia, PA: Elsevier Saunders; chap 141, 2016.

[4] A. S. Hindal, H. A. L. Mossa and M. S. Abood, "Reactive Oxygen Species Levels in Seminal Plasma in a Sample of Iraqi Infertile Men using Advanced Stimulatory Method for Activation of Spermatozoa," International Journal of Medical Research \& Health Sciences, 7(12): 51 - 55, 2018.

[5] E. Ali, U. AL-Kawaz and M. B. Fakhrildin, "A New sperm Preparation Technique by Combination of Density Gradient Centrifugation and Glass Wool Filtration," International Journal of Advanced Research, 4(4):1112-1115, 2004

[6] World Health Organization, "Laboratory Examination and Processing of Human Semen," 5th edition, 2010.

[7] M. Punab, O. Poolamets and P. Paju, "Causes of male infertility: a 9year prospective monocentre study on 1737 patients with reduced total sperm counts," Hum Reprod 32: 18-31, 2017.

[8] Y. Terada, G. Schatten, H. Hasegawa, and N. Yaegashi, "Essential roles of the sperm centrosome in human fertilization: developing the therapy for fertilization failure due to sperm centrosomal dysfunction," The Tohoku Journal of Experimental Medicine, 220(4), 247-258, 2010.

[9] C. J. De Jonge, "Sperm preparation for IVF and ICSI," In Handbook of IVF, $4^{\text {th }}$ edition, $145-156,2017$.

[10] A. A. Kadhim, H. A. Mossa and M. O. A. Selman, "New Sperm Preparation Technique by Glass Wool Filtration Combined with Pentoxifylline Techniques versus Glass Wool Filtration alone for Infertile and Fertile Men," Iraqi Journal of Embryos and Infertility Researches 7(1): 28-36, 2017.

[11] Z. P. Nagy, A. C. Varghese and A. Agarwal, "Practical Manual of In Vitro Fertilization: Advanced Methods and Novel Devices," Springer Science\& Business Media, 2012.

[12] A. A. Kadhim, H. A. Mossa and M. S. Abbood, "A comparison of new sperm preparation technique by glass wool filtration combined with pentoxifylline with other techniques in asthenozoospermic men," International Journal of Advanced Research, 5(4): 1178-1182, 2017.

[13] N. A. Hamza, M. O. Selman and H. A. Mossa, "Comparison of Best Yield of in vitro Sperm activation Techniques with New technique of Caffeine Combined with Density Gradient Centrifugation in Iraqi Patients," Journal of Pharmaceutical Sciences and Research, 10(1): 36-39, 2018.

[14] S. G. Boswell, B. J. Cole, E. A. Sundman, V. Karas and L. A. Fortier, "Platelet-rich plasma: a milieu of bioactive factors," Arthroscopy, 28:429-439, 2012.

[15] L. F. Marques, T. Stessuk, I. C. Camargo, N. Sabeh Junior, L. dos Santos and J. T. Ribeiro-Paes, "Platelet-rich plasma (PRP): methodological aspects and clinical applications," Platelets, 26:101$113,2015$.

[16] M. D. Wild and W. E. Roudebush, "Platelet-activating factor improves intrauterine insemination outcome," Am J Obstet Gynecol, 184:1064-1065, 2001.

[17] B. S. Minhans, R. Kumar, D. D. Ricker, J. L. Robertson and M. G. Dodson, "The presence of platelet activating factor- like activity in human spermatozoa," Fertil Steril, 55(2):372-6, 1991. 